\title{
First experience of junior surgeons with laparoscopic distal gastrectomy: in view of comparison with experienced surgeons
}

\author{
Ki Bum Park ${ }^{1}$, Yoontaek Lee², Dong-Wook Kim³ \\ ${ }^{1}$ Department of Surgery, School of Medicine, Kyungpook National University, Daegu, Korea \\ ${ }^{2}$ Department of Surgery, Ewha Womans University, Seoul Hospital, Seoul, Korea \\ ${ }^{3}$ Department of Surgery, Dankook University, College of Medicine, Cheonan, Korea \\ Videosurgery Miniinv 2021; 16 (1): 123-128 \\ DOI: https://doi.org/10.5114/wiitm.2020.99310
}

\begin{abstract}
Introduction: Laparoscopic surgery is not easily performed by junior surgeons who have limited experience.

Aim: To investigate the safety and feasibility of the first experience of junior surgeons with laparoscopic distal gastrectomy $(L D G)$ who were trained in super high-volume centers.

Material and methods: Clinicopathological data from the first 85 LDG cases performed by three gastric cancer surgeons were collected. All three surgeons were trained for $>1$ year in super high-volume centers. The surgical and postoperative outcomes of the first experiences of junior surgeons were compared with the short-term outcomes reported in a multicenter randomized controlled trial (Korean Laparoendoscopic Gastrointestinal Surgery Study, KLASS-01 trial), conducted by the KLASS group, which is composed of experienced surgeons who practice in a high-volume center.

Results: A significantly greater number of older patients with longer operation times and lower estimated blood loss was observed for the junior surgeons than in the KLASS data. Although junior surgeons performed significantly more Billroth II anastomoses with D1+ lymph node dissection, there was no difference between the two groups in terms of hospital stay, number of retrieved lymph nodes, or postoperative morbidity.

Conclusions: The surgical outcomes of early gastric cancer managed by laparoscopic surgery performed by welltrained beginners were similar to the outcomes reported in the large-scale trial. Therefore, with regard to the surgical training system, training at super high-volume centers may be considered to provide some assurance in terms of surgical technique-related safety.
\end{abstract}

Key words: laparoscopy, surgeons; gastric cancer, learning curve.

\section{Introduction}

Laparoscopic surgery, a minimally invasive surgery, has gained rapid and wide acceptance for the treatment of gastric cancer [1, 2]. Moreover, recent studies demonstrate that short-term outcomes have improved as laparoscopic surgical approaches advance; advances such as reduced-port or single-port procedures reflect the feasibility and safety of laparoscopic surgery [3-5]. Laparoscopic distal gastrectomy (LDG) has certain advantages in terms of wound complication, quality of life, and cosmetic aspects $[6,7]$. In a recent Korean Laparoendoscopic Gastrointestinal Surgery Study Group (KLASS)-01 study, oncologic safety was also proven to be an advantage of LDG, one not held by open procedures [8]. According to the Korean guidelines published in 2019, LDG is recommended as the treatment of 
choice for patients with early gastric cancer (EGC) [9]. Indeed, the era of open gastrectomy is gradually coming to an end for EGC patients, and this is the era of laparoscopy.

However, laparoscopic surgery is not easily performed by junior surgeons who have limited experience $[10,11]$. The majority of junior surgeons work in low-volume centers, which do not receive many operative cases. Hence, junior surgeons trained at these low-volume centers require considerable time to become adept in laparoscopic surgery; this results in a steep learning curve. Surgeons must be familiar with various laparoscopic instruments and the laparoscopic view, which is somewhat different from the visual field of a laparotomy. In addition, laparoscopy requires greater ability of assistants (including first assistant, scopist) than open surgery. The difficulty of securing a skilled surgical team at a low-volume center is thought to impact the rate at which these junior surgeons hone the skills required for laparoscopic surgery.

\section{Aim}

This study aims to investigate the safety and feasibility of the first LDG of junior surgeons trained in super high-volume centers that granted them adequate caseloads and training in laparoscopic operations. The short-term outcomes of LDG between three junior surgeons with 3 to 4 years of experience in the gastrointestinal division of department of surgery were compared with the outcomes of the study conducted by the KLASS group, which is composed of experienced surgeons who practice in a high-volume center. We hypothesized that junior surgeons who trained in a super high-volume center can perform LDG without much strain. We evaluated the factors that allow surgeons at the beginning of their careers to perform LDG safely for the treatment of patients with EGC.

\section{Material and methods}

\section{Patients and data collection}

Three junior gastric cancer surgeons who were trained for 1-2 years in a super high-volume center before moving to their current workplace were recruited in this study. During their training period, junior surgeons performed about 20 cases of total LDG operations and about 300 cases of partial procedures, including gastrointestinal anastomosis.
Data from approximately 30 initial LDG cases for cT1 gastric cancer were collected from each surgeon for the study between March 2018 and August 2019. Curative distal gastrectomy and standard lymph node dissection including partial omentectomy were performed for EGC. A Billroth II reconstruction procedure or Roux-en-Y gastrojejunostomy was performed intracorporeally using a linear stapler according to the surgeon's preference and patient condition. Clinicopathologic features of the enrolled patients, including age, sex, body mass index (BMI), American Society of Anesthesiologists (ASA) score, operative time, tumor stage, number of retrieved lymph nodes, and postoperative complications were collected retrospectively from the electronic medical records of each surgeon's hospital. The pathologic stage was classified according to the $8^{\text {th }}$ edition of the American Joint Cancer Committee tumor, node, and metastasis (TNM) classification system [12]. Postoperative complications occurring within 30 days of surgery were evaluated according to the Clavien-Dindo classification [13]. We compared our data with the prospective randomized controlled trial of the KLASS group [7].

This study was approved by the institutional review boards (IRB) of the Ethics Committees at each of the enrolled institutions (approval number; 201907-007 at the institution of the first author). Patient records were anonymized and deidentified before analysis. Written informed consent was waived by the IRB. This study was conducted in accordance with the Helsinki Declaration as revised in 2013.

\section{Statistical analysis}

Continuous variables are presented as mean ( \pm standard deviation). Statistical analyses were performed using the one-sample $t$-test for continuous variables, and the $\chi^{2}$ test for categorical variables. A $p$-value threshold of 0.05 was considered statistically significant. All statistical analyses were performed with R software (R Foundation for Statistical Computing, Vienna, Austria; http://cran.r-project. org/).

\section{Results}

Eighty-five patients who had undergone LDG performed by three junior surgeons were included in the present study. We compared these data to 644 patients of the per protocol group in the KLASS-01 
trial [7]. The clinicopathological characteristics of the patients are shown in Table I. The patients in the junior surgeons' group were older than those in the KLASS group (63.4 \pm 10.4 vs. $56.8 \pm 10.9, p<0.001)$. There was no difference in sex, BMI, ASA scores, or tumor size between the two groups.

Regarding surgical outcomes, the junior surgeon group was associated with significantly longer operation times $(209.9 \pm 51.4$ vs. $184.1 \pm 53.3 \mathrm{~min}$, $p<0.001)$ and lower intraoperative blood loss (51.5 \pm 45.9 vs. $190.6 \pm 156.3 \mathrm{ml}, p<0.001)$. Although junior surgeons performed significantly more Billroth II anastomosis with D1+ lymph node dissections $(p<0.001)$, there was no difference between the two groups in hospital stay $(7.2 \pm 3.1$ vs. $7.1 \pm 3.1$ days, $p=0.780$ ), number of retrieved lymph nodes (39.3 \pm 14.7 vs. $40.5 \pm 15.3, p=0.495)$, or postoperative morbidities ( $9.0 \%$ vs. $13.0 \%, p=0.115$ ) (Table II).

In 6 morbidity cases, 1 patient corresponded to a Clavien-Dindo grade of greater than II (Table III). This patient had symptoms of intestinal obstruction on postoperative day four after an LDG with Billroth II anastomosis. Diagnostic laparoscopy was performed

Table I. Comparison of clinicopathological characteristics

\begin{tabular}{|lccc|}
\hline Parameter & $\begin{array}{c}\text { Junior } \\
(n=85)\end{array}$ & $\begin{array}{c}\text { KLASS } \\
(n=644)\end{array}$ & $P$-value \\
\hline Age [years] & $63.4 \pm 10.4$ & $56.8 \pm 10.9$ & $<0.001$ \\
\hline \begin{tabular}{l} 
Sex: \\
\hline Female
\end{tabular} & $23(27.1 \%)$ & $219(34.0 \%)$ & 0.201 \\
\hline Male & $62(72.9 \%)$ & $425(66.0 \%)$ & \\
\hline $\begin{array}{l}\text { Body mass } \\
\text { index [kg/m²] }\end{array}$ & $24.2 \pm 3.3$ & $23.8 \pm 2.9$ & 0.240 \\
\hline $\begin{array}{l}\text { ASA score: } \\
1\end{array}$ & $36(42.4 \%)$ & $319(59.5 \%)$ & \\
\hline 2 & $5(51.8 \%)$ & $291(45.2 \%)$ & \\
\hline$\geq 3$ & $2.7 \pm 2.1$ & $2.71 \pm 1.6$ & 0.966 \\
\hline Tumor size $[\mathrm{cm}]$ & $34(5.3 \%)$ & 0.006 \\
\hline Stage & $77(90.6 \%)$ & $487(75.6 \%)$ & \\
\hline IA & $7(8.2 \%)$ & $104(16.1 \%)$ & \\
\hline IB & $1(1.2 \%)$ & $53(8.2 \%)$ & \\
\hline II or more & & & \\
\hline
\end{tabular}

Data shown are number (\%), mean (SD). KLASS - Korean Laparoendoscopic Gastrointestinal Surgery Study, ASA - American Society of Anesthesiologists, SD - standard deviation. and an internal herniation was identified in the mesenteric defect of the jejunojejunostomy site. After the laparoscopic hernia reduction, the patient fully recovered.

\section{Discussion}

A surgeon's experience is one of the most important factors for reducing postoperative morbidity and mortality $[14,15]$. From the perspective of a novice, it is true that surgical techniques cannot be mastered by just studying textbooks or viewing operation videos. Surgeons usually build their experience by watching and imitating surgery performed by an experienced surgeon who is called a mentor; surgeons begin to operate on their own when they reach a certain stage. However, it is not easy to gain surgical experience over a short period of time. In the case of gastric cancer, operation numbers are gradually decreasing due to the downward course of pathogenesis and recent advances of endoscopic procedures to treat EGC [16]. Moreover, most of the operations are performed in

Table II. Surgical outcomes

\begin{tabular}{|c|c|c|c|}
\hline Parameter & $\begin{array}{l}\text { Junior } \\
(n=85)\end{array}$ & $\begin{array}{c}\text { KLASS } \\
(n=644)\end{array}$ & $P$-value \\
\hline Operation time [min] & $209.9 \pm 51.4$ & $184.1 \pm 53.3$ & $<0.001$ \\
\hline $\begin{array}{l}\text { Estimated blood } \\
\text { loss [ml] }\end{array}$ & $51.5 \pm 45.9$ & $190.6 \pm 156.3$ & $<0.001$ \\
\hline Combined resection & $4(4.7 \%)$ & $20(3.1 \%)$ & 0.437 \\
\hline LN dissection: & & & $<0.001$ \\
\hline D1+ & $71(83.5 \%)$ & $284(44.3 \%)$ & \\
\hline D2 & $14(16.5 \%)$ & 360 (55.7\%) & \\
\hline Reconstruction: & & & $<0.001$ \\
\hline Billroth-I & $0(0 \%)$ & $413(64.1 \%)$ & \\
\hline Billroth-II & $77(90.6 \%)$ & $222(34.5 \%)$ & \\
\hline Roux-en-Y & $8(9.4 \%)$ & $9(1.4 \%)$ & \\
\hline $\begin{array}{l}\text { Number of retrieved } \\
\text { LNs }\end{array}$ & $39.3 \pm 14.7$ & $40.5 \pm 15.3$ & 0.495 \\
\hline $\begin{array}{l}\text { Postoperative } \\
\text { hospital stay [days] }\end{array}$ & $7.2 \pm 3.1$ & $7.1 \pm 3.1$ & 0.780 \\
\hline $\begin{array}{l}\text { Complications } \\
\text { within } 30 \text { days }\end{array}$ & $6(9.0 \%)$ & $84(13.0 \%)$ & 0.115 \\
\hline
\end{tabular}

Data shown are number (\%), mean (SD). KLASS - Korean Laparoendoscopic Gastrointestinal Surgery Study, LN - lymph node, SD - standard deviation. 
Table III. Morbidity cases

\begin{tabular}{|c|c|c|c|c|c|c|c|}
\hline Sex & Age & $\mathrm{BMI}\left[\mathrm{kg} / \mathrm{m}^{2}\right]$ & ASA score & $\begin{array}{c}\text { Type } \\
\text { of complication }\end{array}$ & $\begin{array}{l}\text { Clavien-Dindo } \\
\text { grade }\end{array}$ & Treatment & $\begin{array}{l}\text { Full } \\
\text { recovery }\end{array}$ \\
\hline M & 62 & 22.9 & 2 & Internal hernia & IV & $\begin{array}{c}\text { Operation } \\
\text { (hernia reduction) }\end{array}$ & Yes \\
\hline $\mathrm{F}$ & 80 & 22.1 & 2 & C. difficile colitis & ॥ & Antibiotics & Yes \\
\hline M & 58 & 23.4 & 2 & Pneumonia & ॥ & Antibiotics & Yes \\
\hline M & 57 & 22.0 & 2 & $\begin{array}{l}\text { Wound complica- } \\
\text { tion }\end{array}$ & । & Conservative treatment & Yes \\
\hline M & 48 & 26.0 & 1 & Ileus & ॥ & Conservative treatment & Yes \\
\hline M & 78 & 19.5 & 2 & Ileus & $\|$ & Conservative treatment & Yes \\
\hline
\end{tabular}

$B M I$ - body mass index, ASA - American Society of Anesthesiologists, $M$ - male, $F$-female.

certain super high-volume centers in metropolitan areas; hence, there are not many opportunities for junior surgeons who work in small local hospitals to perform gastrectomies.

Laparoscopic gastrectomy is a complex procedure that includes lymph node dissection and various intestinal anastomoses $[17,18]$, requiring a considerable learning curve to overcome $[1,15]$. The learning curve, the definition of which is clearly arbitrary, depends on factors such as the talent of surgeons, quality of teaching programs, and amount of experience of the surgeons $[19,20]$. The learning curve of LDG is reported to be around 50 cases [21, 22]. In the present study, the junior surgeons performed about 20 complete cases of LDG operations and about 300 cases of partial procedures during their training period. Intensive training in super high-volume centers can be an effective way for junior surgeons to overcome the learning curve of laparoscopic gastrectomy [23]. As a result, the junior surgeons in this study were capable of overcoming the learning curve after the training. In Korea, there are some super high-volume centers with cutting edge infrastructure, at which over 1,000 gastrectomies are performed annually. In his editorial, Kodera expresses his envy of this type of center as an educational environment for young surgeons [24]. As the present study compared the short-term results of LDG in patients with EGC performed by three junior surgeons who trained in a super high-volume center, with the short-term results of a group of experienced surgeons that represents KLASS in Korea, we consider the following points for discussion.

The results of this study are intended to examine the surgical environment and situation of junior surgeons. First, junior surgeons tend to operate on older patients. Due to the medical characteristics of Korea, many patients visit experienced doctors and tend to flock to high volume centers. Older patients, however, may be more likely to go to local doctors rather than to large hospitals; older patients tend to choose a doctor who is more accessible rather than search for a more experienced doctor. Second, in LDG performed by junior surgeons, the operation time tends to be long while estimated blood loss is low. Shortening of long operation times is a component of the learning curve present at the beginning of a surgical career. The junior surgeons had not yet adapted to the new operating room, instruments and surgical team in their new hospitals, which contributed to the longer surgery times [24]. In addition, junior surgeons operate slowly in an effort to adhere to the surgical guidelines. The poor quality and low quantity of assistants would have lengthened operating times as well. The lower estimated blood loss may be an inaccurate measurement of retrospective data in the group of junior surgeons.

The results pertaining to intestinal anastomosis were of particular interest. In our study, Billroth I anastomosis, which generally requires the aid of a surgical assistant, was not performed. Most junior surgeons preferred Billroth II anastomosis, which does not require the role of a surgical assistant. Junior surgeons often do not have surgical assistants at the time of surgery. This can be explained by the lack of surgical residents in general surgery programs and the low positions of influence held by junior surgeons in each hospital. Through training under such a structure, many junior surgeons have grown familiar with performing surgery without 
assistance. It is in line with this trend that interest and research regarding reduced-port surgery or solo surgery has risen within the junior surgeon communities.

Although the surgical environment differed between the junior surgeons and the experienced surgeons, no difference in postoperative complications or mortality was noted in our study data. Training in super high-volume centers allows for more opportunities to participate in the operating room. Super high-volume centers also provide the opportunity to gain experience in the management of a diverse array of complications that may occur post-operatively. All three junior surgeons were trained by the same mentors at one of the representative super high-volume centers in Korea. They are mentors of laparoscopic surgery who are a pioneer of laparoscopic gastrectomy in Korea with the principal investigator of the KLASS-01 study, an expert of single-port gastrectomy with the principal investigator of the KLASS-05 study, and a representative of solo-single-port gastrectomy, respectively. Under these mentors, the junior surgeons' training benefitted from various surgical and academic activities. In addition, this hospital conducts nearly 1,000 gastrectomies per year, which is made possible by an optimized operation system including a specialized surgical team with a skillful first assistant, experienced scopists, and scrub nurses. Even now, many beginners who want to be decent laparoscopic surgeons are waiting to be trained in this hospital.

This study has some limitations. First, it is a retrospective analysis with a small sample size which might have been biased by several factors. Second, the timing of the surgery is different. The KLASS study was performed from 2006 to 2010, which is about 10 years prior to the time of our study. Within a decade, laparoscopic technology has evolved and includes tools such as three-dimensional scopes, automatic linear staplers, and new suture materials. These facts could have affected our analysis. Third, the definition of a super high-volume center is ambiguous, and each center may have different educational protocols and opportunities for individuals; the diversity of protocols interferes with the ability to generalize our conclusions. Finally, long-term results of patients were not obtained because of the short follow-up time. Oncologic safety should be considered a necessity when describing the safety of cancer operations.

\section{Conclusions}

A surgeon becomes adept by accumulating experience, which can be through direct and indirect methods. In reality, it is almost impossible to obtain all the experience by ourselves. To increase exposure to operations, it is important to gain experience from a well-skilled and experienced surgeon. Our study is the first to compare the surgical outcomes of junior surgeons trained in a super high-volume center with the large scale data of experienced surgeons. We could cautiously conclude that junior surgeons perform LDG with safety and feasibility in the treatment of patients with EGC. However, this conclusion does not imply that junior surgeons must be trained in super high-volume centers, but that training in super high-volume centers allows junior surgeons to perform surgery skillfully and safely in a short time; thus, with regard to the surgical training system, training at super high-volume centers may be considered to provide some assurance in terms of surgical technique-related safety.

\section{Acknowledgments}

The authors wish to thank three mentors Pf. Hyung-Ho Kim, Do Joong Park, and Sang-Hoon Ahn at Seoul National University Bundang Hospital.

\section{Conflict of interest}

The authors declare no conflict of interest.

\section{References}

1. Kim HH, Han SU, Kim MC, et al. Long-term results of laparoscopic gastrectomy for gastric cancer: a large-scale case control and case-matched Korean multicenter study. J Clin Oncol 2014; 32: 627-33.

2. Kim YW, Baik YH, Yun YH, et al. Improved quality of life outcomes after laparoscopy assisted distal gastrectomy for early gastric cancer: results of a prospective randomized clinical trial. Ann Surg 2008; 248: 721-7.

3. Kim SM, Ha MH, Seo JE, et al. Comparison of reduced port totally laparoscopic distal gastrectomy (duet TLDG) and conventional laparoscopic-assisted distal gastrectomy. Ann Surg Oncol 2015; 22: 2567-72.

4. Ahn SH, Son SY, Lee CM, et al. Intracorporeal uncut Roux-en-Y gastrojejunostomy reconstruction in pure single-incision laparoscopic distal gastrectomy for early gastric cancer: unaided stapling closure. J Am Coll Surg 2014; 218: 17-21.

5. Jeong O, Park YK, Ryu SY. Early experience of duet laparoscopic distal gastrectomy (duet-LDG) using three abdominal ports for gastric carcinoma: surgical technique and comparison with 
conventional laparoscopic distal gastrectomy. Surg Endosc 2016; 30: 3559-66

6. Katai H, Mizusawa J, Katayama $\mathrm{H}$, et al. Short-term surgical outcomes from a phase III study of laparoscopy-assisted versus open distal gastrectomy with nodal dissection for clinical stage IA/IB gastric cancer: Japan Clinical Oncology Group Study JCOG0912. Gastric Cancer 2017; 20: 699-708.

7. Kim W, Kim HH, Han SU, et al. Decreased morbidity of laparoscopic distal gastrectomy compared with open distal gastrectomy for stage I gastric cancer: Short-term outcomes from a multicenter randomized controlled trial (KLASS-01). Ann Surg 2016; 263: 28-35.

8. Kim HH, Han SU, Kim MC, et al. Effect of laparoscopic distal gastrectomy vs open distal gastrectomy on long-term survival among patients with stage I gastric cancer. JAMA Oncol 2019; 5: 506-13.

9. Korean Practice Guideline for Gastric Cancer 2018: an evidence-based, multi-disciplinary approach. J Gastric Cancer 2019; 19: 1-48.

10. Jung do H, Son SY, Park YS, et al. The learning curve associated with laparoscopic total gastrectomy. Gastric Cancer 2016; 19: 264-72.

11. Kim MG, Kim KC, Yook JH, et al. A practical way to overcome the learning period of laparoscopic gastrectomy for gastric cancer. Surg Endosc 2011; 25: 3838-44.

12. Amin MB, Edge SB, Greene FL, et al. (eds): AJCC Cancer Staging Manual. $8^{\text {th }}$ ed. Springer, New York 2017.

13. Clavien PA, Barkun J, de Oliveira ML, et al. The Clavien-Dindo classification of surgical complications: five-year experience. Ann Surg 2009; 250: 187-96.

14. Liang Y, Wu L, Wang X, et al. The positive impact of surgeon specialization on survival for gastric cancer patients after surgery with curative intent. Gastric Cancer 2015; 18: 859-67.

15. Lee HH, Son SY, Lee JH, et al. Surgeon's experience overrides the effect of hospital volume for postoperative outcomes of laparoscopic surgery in gastric cancer: multi-institutional study. Ann Surg Oncol 2017; 24: 1010-7.

16. Information Committee of Korean Gastric Cancer A. Korean Gastric Cancer Association nationwide survey on gastric cancer in 2014. J Gastric Cancer 2016; 16: 131-40.

17. Choi M, Ko CS, Yook JH, et al. Comparative outcomes between totally laparoscopic total gastrectomy with the modified overlap method for early gastric cancer and advanced gastric cancer: review of 149 consecutive cases. Videosurgery Miniinv 2020; 15: 437-45.

18. Zheng CY, Dong ZY, Zheng LZ, et al. Laparoscopic D2 plus complete mesogastrium excision using the "enjoyable space" approach versus conventional D2 total gastrectomy for local advanced gastric cancer: short-term outcomes. Videosurgery Miniinv 2020; 15: 58-69.

19. Brzoszczyk B, Milecki T, Jarzemski P, et al. Urology resident training in laparoscopic surgery - results of the first national survey in Poland. Videosurgery Miniinv 2019; 14: 433-41.

20. Cumpanas AA, Bardan R, Ferician O, et al. The impact of tiredness on virtual reality robotic surgical skills. Videosurgery Miniinv 2020; 15: 298-304.
21. Zhao LY, Zhang WH, Sun Y, et al. Learning curve for gastric cancer patients with laparoscopy-assisted distal gastrectomy: 6-year experience from a single institution in western China. Medicine 2016; 95: e4875.

22. Moon JS, Park MS, Kim JH, et al. Lessons learned from a comparative analysis of surgical outcomes of and learning curves for laparoscopy-assisted distal gastrectomy. I Gastric Cancer 2015; 15: 29-38.

23. Jee YS. First experiences with laparoscopic assisted distal gastrectomy: in the view of comparison with high volume centers. J Korean Surg Soc 2012; 83: 130-4.

24. Kodera Y. Extremity in surgeon volume: Korea may be the place to go if you want to be a decent gastric surgeon. Gastric Cancer 2016; 19: 323-5.

Received: 28.07.2020, accepted: 24.08.2020. 